\title{
Pentoxifylline Enhances the Radioprotective Properties of $\mathrm{Y}$ - Tocotrienol: Differential Effects on the Hematopoietic, Gastrointestinal and Vascular Systems
}

\author{
Maaike Berbée ${ }^{a, 1}$, Qiang Fu ${ }^{a}$, Sarita Garg ${ }^{a}$, Shilpa Kulkarni ${ }^{b}$, K. Sree Kumar ${ }^{b}$, and Martin \\ Hauer-Jensen ${ }^{\mathrm{a}, \mathrm{c}, 2}$ \\ a Division of Radiation Health, University of Arkansas for Medical Sciences, Little Rock, Arkansas \\ b Armed Forces Radiobiology Research Institute, Uniformed Services University, Bethesda, \\ Maryland ${ }^{\mathrm{C}}$ Surgical Service, Central Arkansas Veterans Healthcare System, Little Rock, \\ Arkansas
}

\begin{abstract}
The vitamin E analog $\gamma$-tocotrienol (GT3) is a potent radioprotector and mitigator. This study was performed to (a) determine whether the efficacy of GT3 can be enhanced by the addition of the phosphodiesterase inhibitor pentoxifylline (PTX) and (b) to obtain information about the mechanism of action. Mice were injected subcutaneously with vehicle, GT3 [400 mg/kg $24 \mathrm{~h}$ before total-body irradiation (TBI)], PTX (200 $\mathrm{mg} / \mathrm{kg} 30 \mathrm{~min}$ before TBI), or GT3+PTX before being exposed to $8.5-13$ Gy TBI. Overall lethality, survival time and intestinal, hematopoietic and vascular injury were assessed. Cytokine levels in the bone marrow microenvironment were measured, and the requirement for endothelial nitric oxide synthase (eNOS) was studied in eNOSdeficient mice. GT3+PTX significantly improved survival compared to GT3 alone and provided full protection against lethality even after exposure to $12.5 \mathrm{~Gy}$. GT3+PTX improved bone marrow CFUs, spleen colony counts and platelet recovery compared to GT3 alone. GT3 and GT3+PTX increased bone marrow plasma G-CSF levels as well as the availability of IL-1a, IL-6 and IL-9 in the early postirradiation phase. GT3 and GT3+PTX were equally effective in ameliorating intestinal injury and vascular peroxynitrite production. Survival studies in eNOS-deficient mice and appropriate controls revealed that eNOS was not required for protection against lethality after TBI. Combined treatment with GT3 and PTX increased postirradiation survival over that with GT3 alone by a mechanism that may depend on induction of hematopoietic stimuli. GT3+PTX did not reduce GI toxicity or vascular oxidative stress compared to GT3 alone. The radioprotective effect of either drug alone or both drugs in combination does not require the presence of eNOS.
\end{abstract}

\section{INTRODUCTION}

Pharmacological strategies to prevent or reduce radiation-induced injury and lethality after total-body irradiation (TBI) are urgently needed. Recent studies have identified the vitamin $\mathrm{E}$ analog $\gamma$-tocotrienol (GT3) as a powerful radioproprotective agent with a dose reduction factor (DRF) in excess of 1.3 as well as a postexposure mitigating agent when administered alone (1-3). Over the years, however, several groups have reported that combined treatment with vitamin $\mathrm{E}$ and the methylxanthine derivative pentoxifylline (PTX) may be particularly

\footnotetext{
${ }^{2}$ Address for correspondence: Division of Radiation Health, University of Arkansas for Medical Sciences, 4301 West Markham, Slot 522-10, Little Rock, AR 72205; mhjensen@life.uams.edu.

${ }_{1}$ Current address: Department of Radiation Oncology (Maastro), GROW Research Institute, Maastricht University Medical Center,

Maastricht, The Netherlands.
} 
effective in reducing tissue injury after localized irradiation. For example, vitamin $\mathrm{E}$ combined with PTX has been shown in both preclinical and clinical studies to significantly reduce and in some circumstances even reverse radiation-induced cardiac, lung, intestinal and dermal injury (4-9). However, most previous studies have assessed the effects of vitamin E and PTX on radiation-induced fibrosis, a late complication of radiation exposure, while little is known about the effects of this combination on acute radiation injury and radiation-induced mortality.

The radioprotective effects of GT3 depend not only on its antioxidative properties but also on its abilities to concentrate in endothelial cells and inhibit the enzyme 3-hydroxy-3methylglutaryl-coenzyme A (HMG-CoA) reductase, similar to the drug class statins. HMGCoA reductase inhibitors are commonly used in the treatment of hyperlipidemia disorders but in addition have a plethora of vasculoprotective, anti-inflammatory and anti-fibrotic effects mediated by endothelial nitric oxide synthase (eNOS, NOS3) $(10,11)$. While it acts through different and partly unknown mechanisms, PTX has similar antioxidant, vasculoprotective, anti-inflammatory and anti-fibrotic properties and similarly increases eNOS activity through an increase in intracellular cyclic adenosine monophosphate (cAMP) (12-14).

In this study, we examined the effects of GT3 in combination with PTX on TBI-induced acute hematopoietic, intestinal and vascular injury and subsequent mortality. We further used eNOS-deficient mice to determine whether protection against lethality from either drug alone or the combination required the presence of eNOS. Combined therapy was significantly more effective in improving postirradiation survival than treatment with GT3 only, but the effect on postirradiation lethality did not require the presence of eNOS.

Moreover, our data suggest that administration of GT3 together with PTX may modulate the hematopoietic radiation response by the induction of hematopoietic stimuli. GT3 combined with PTX also reduced postirradiation intestinal injury and vascular oxidative stress compared to vehicle, but no additional benefit was observed by the addition of PTX to GT3 compared to treatment with GT3 alone.

\section{MATERIALS AND METHODS}

\section{Chemicals}

GT3 was obtained from Yasoo Health Inc. (Johnson City, TN). Shortly before administration, GT3 was dispersed in a mixture of polyethylene glycol (PEG-400) (Sigma, St. Louis, MO) and a proprietary emulsifying agent (Stuart Products, Bedford, TX). PEG-400 with the emulsifying agent but without GT3 was used as a vehicle control. PTX was obtained from Sigma and dissolved in saline. Saline without PTX was used as a vehicle control. Unless otherwise specified, all other chemicals were obtained from Sigma.

\section{Animals}

The experimental protocol was reviewed and approved by the Central Arkansas Veterans Healthcare System (CAVHS) Institutional Animal Care and Use Committee (IACUC). Male CD2F1 mice (Harlan Sprague Dawley, Indianapolis, IN) with a body weight of 22-25 g were used for comparison of hematopoietic, intestinal and vascular radiation responses. For the experiments to investigate the requirement for eNOS in lethality protection, B6.129P2Nos $3^{\text {tm } 1 \text { Unc } / J ~ b r e e d i n g ~ p a i r s ~ w e r e ~ o b t a i n e d ~ f r o m ~ t h e ~ J a c k s o n ~ L a b o r a t o r y ~(B a r ~ H a r b o r, ~ M E) ~}$ and bred in our breeding facility. In this study, eNOS-deficient male offspring 6-8 weeks old were used. Age- and sex-matched C57BL/6 mice (Charles River, Wilmington, MA) were used as wild-type controls. 
Animals were housed in conventional cages under standardized conditions with controlled temperature and humidity and a 12-12-h day-night light cycle. Animals had free access to water and chow (Harlan Teklad laboratory diet 7012, Purina Mills, St. Louis, MO).

A total of 368 mice were used for these experiments, with 4-8 mice per group. Mice were randomly assigned to one of the four following treatment groups: vehicle control, PTX alone, GT3 alone, and GT3 with PTX. Twenty-four hours before irradiation, mice received a single dose of GT3 $(400 \mathrm{mg} / \mathrm{kg})$ or the excipient alone by s.c. injection. Thirty minutes before irradiation, mice received a single dose of PTX $(200 \mathrm{mg} / \mathrm{kg})$ or saline solution by s.c. injection. These doses and administration schedules (GT3, 200-400 mg/kg, administered 22-24 h before TBI; PTX, $200 \mathrm{mg} / \mathrm{kg}$, administered 15-30 min before TBI) had been determined to confer optimal lethality protection and were arrived at through extensive range-finding studies performed in parallel at two separate institutions (University of Arkansas for Medical Sciences and Armed Forces Radiobiology Research Institute) prior to initiating the experiments reported here.

To study the effect of PTX, GT3 and GT3+PTX on postirradiation survival, mice received 10.5, 11.5 and 12.5 Gy TBI and were observed for 30 days.

To determine the effect of the different treatments on radiation-induced intestinal and hematopoietic injury, mice received a single dose of TBI ( $8.5 \mathrm{~Gy}$, unless otherwise specified) and were subsequently killed humanely at set times after irradiation $(0 \mathrm{~h} / \mathrm{no}$ irradiation, 1 day, 3.5 days, 7 days, 10 days, 14 days). Previous experiments in CD2F1 mice have shown that 8.5 Gy TBI induces pronounced intestinal and hematopoietic injury together with relatively low 14-day mortality.

\section{Irradiation}

Unanesthetized mice were exposed to a single whole-body radiation dose in a Shepherd Mark I, model $25{ }^{137}$ Cs irradiator (J. L. Shepherd \& Associates, San Fernando, CA). During irradiation, the mice were placed in a well-ventilated cylindrical chamber made specifically for irradiation of mice (J.L. Shepherd \& Associates). The chamber was made of Plexiglas and was divided into four $90^{\circ}$ "pie slice" compartments by vertical dividers made of T-6061 aluminum (machinable grade) with a gold anodized coating. The average dose rate was 1.35 Gy per min and was corrected for decay each day mice were irradiated.

\section{Survival Studies}

To study postirradiation survival, mice received $10.5,11.5$ or 12.5 Gy TBI. Mice were monitored for 30 days, and the number of dead/moribund mice was recorded twice daily. Kaplan-Meier survival curves and median survival times were calculated.

\section{Assessment of Intestinal Radiation Injury}

1. Mucosal surface area (MSA) - Intestinal mucosal surface area is a well-validated, sensitive parameter of intestinal radiation injury. Mucosal surface area was measured in vertical H\&E-stained sections of the jejunum using a projection/cycloid method as described by Baddeley et al. (15). The method has previously been validated specifically for surface area determination of the intestinal mucosa after irradiation (16).

2. Intestinal crypt colony assay-Microcolony crypt cell survival was assayed as described by Withers and Elkind (17). At 3.5 days after TBI (0, 8.5, 11, 13 and 15 Gy), mice were killed and segments of proximal jejunum were obtained, fixed and H\&E-stained. Surviving crypts, defined as crypts containing 10 or more adjacent chromophilic non-Paneth cells, were counted in transverse cross sections. Four circumferences were scored per mouse 
and microcolony survival was expressed as the average number of crypts per circumference, with the average from each mouse considered as a single value for statistical purposes.

\section{Assessment of Hematopoietic Injury}

1. Bone marrow plasma and bone marrow CFUs-The bone marrow of both femurs was collected from CD2F1 mice at days $0,1,10$ and 14 by rinsing the femurs with $200 \mu 1$ sterile PBS. The cell suspension was centrifuged ( $5 \mathrm{~min}, 3000 \mathrm{rpm}$ ), after which the supernatant was separated from the cell pellet. The supernatant or so-called bone marrow plasma was collected and stored at $-80^{\circ} \mathrm{C}$ until further analysis. The bone marrow plasma was used to obtain information about the bone marrow microenvironment. Bone marrow cytokine/chemokine levels were measured by multiplexing using a Bioplex system (Bio-Rad Laboratories, Hercules, CA) and the BioSource mouse cytokine/chemokine 20-plex panel (Invitrogen, Carlsbad, CA). Cytokine/chemokine levels were expressed per mg total protein. The protein concentration in each sample was measured with a modified Bradford reaction (Coomassie Plus Protein Assay, Thermo Scientific, Rockford, IL) according to the manufacturer's instructions.

Bone marrow cells collected at day 1 postirradiation were used to determine the effect of the different treatments on MB-CFU. Bone marrow cells were suspended in MethoCult methylcellulose medium (Stem Cell Technologies, Vancouver, BC, Canada) at a concentration of $10^{5} \mathrm{cells} / \mathrm{ml}$. The assay was performed in triplicate. A total of $1.1 \mathrm{ml}$ cellmedium suspension was dispensed per 35-mm cell culture dish. Cell colonies were counted after 12 days. For each mouse, the average of the three cell culture dishes was calculated and considered as a single value for statistical purposes.

2. Spleen colonies-Spleen colony counts are a marker of postirradiation hematopoietic recovery. Endogenous spleen colonies are a result of the proliferation of single hematopoietic stem cells. At 10 days after TBI, spleens were collected in Bouin's solution. After fixation, the spleen colonies were clearly visible as yellowish nodules against a dark, smooth background. Spleen colonies were counted by two independent observers.

\section{Blood Cell Counts}

At $0,1,10$ and 14 days after 8.5 Gy TBI, blood was collected in an EDTA-coated tube by retro-orbital puncture. Peripheral blood cell counts were obtained using a veterinary Hematrue system (Heska Corporation, Loveland, $\mathrm{CO}$ ) according to the manufacturer's instructions.

\section{Vascular Peroxynitrite Production}

Vascular peroxynitrite production was measured as described before (1). The abdominal aorta was dissected with as little perivascular tissue as possible. After collection, the aortas were incubated with $10 \mu M$ dihydrorhodamine 123 (DHR123) (Axxora, San Diego, CA) in EGM-2 medium (Lonza, Walkersville, MA) for $90 \mathrm{~min}$ at $37^{\circ} \mathrm{C}$ in the dark. Subsequently, aortas were washed twice with PBS and homogenized in a buffer (PBS, 0.1\% Tween-20, $0.1 \%$ SDS) using a Polytron PT 6100 homogenizer (Kinematica Inc., Bohemia, NY).

Samples were centrifuged for $5 \mathrm{~min}$ at $2000 \mathrm{rpm}$ and supernatant was collected to determine fluorescence (485/515) using a Synergy HT multiplate reader (BioTek Instruments, Winooski, VT).

Protein concentration in the supernatant was measured using a modified Bradford reaction (Coomassie Plus Protein Assay, Thermo Scientific, Rockford, IL). Fluorescence was expressed per mg protein. At each time, data were expressed relative to the vehicle group. 


\section{Statistical Methods}

Sample size estimation and power analysis for the survival experiments were performed as described elsewhere (18). Calculation of the number of animals required for the other end points was performed with PASS (NCSS, Kaysville, UT) for fixed effect analysis of variance (ANOVA) or two-group designs as appropriate, using a significance level of 0.05 and aiming for statistical power of $>80 \%$ in all experiments.

Statistical analyses were performed using NCSS 2004 for Windows (NCSS, Kaysville, UT). Data are presented as means \pm SEM except for duration of survival, which is presented as median \pm interquartile range (IQR). Two-sided tests were used throughout, and differences were considered statistically significant when the $P$ value was less than 0.05 . Survival curves were constructed using the Kaplan-Meier method and were compared using the log-rank test. Mucosal surface areas and vascular peroxynitrite production were compared using regression analysis with time and treatment group as independent variables. Survival curves for the crypt colony assay were compared using regression analysis with radiation dose and treatment group as independent variables. End points from individual animals or observers were averaged and considered as single values for statistical purposes. Pairwise (univariate) comparisons were performed with the Mann-Whitney $U$ test.

\section{RESULTS}

\section{Overall Lethality and Duration of Survival}

The effects of PTX, GT3 and GT3 combined with PTX on postirradiation survival were determined in mice exposed to 10.5, 11.5 and 12.5 Gy. Exposure to radiation induced signs of radiation sickness (e.g. diarrhea, weight loss, lethargy) and mortality. The severity of the symptoms increased with increasing radiation dose. Treatment with GT3 together with PTX significantly improved postirradiation survival in mice treated with 10.5, 11.5 and 12.5 Gy ( $P=0.0001, P<0.0001$ and $P<0.0001$, respectively) (Fig. 1, Table 1). All animals, i.e. animals exposed to $10.5,11.5$ and $12.5 \mathrm{~Gy}$, treated with GT3 in combination with PTX survived the 30-day postirradiation observation period. Treatment with only PTX did not affect postirradiation survival. GT3 decreased postirradiation lethality in animals treated with both $10.5 \mathrm{~Gy}(P=0.001)$ and $11.5 \mathrm{~Gy}(P=0.004)$ but not in animals treated with 12.5 Gy. At the highest dose of 12.5 Gy there was a significance difference in survival between GT3-treated animals and animals treated with GT3 in combination with PTX $(P=0.009)$.

Compared to vehicle, which was associated with $100 \%$ lethality after 8.5 Gy TBI, GT3, PTX and GT3+PTX conferred complete or near-complete protection in both C57BL/6 and eNOS-deficient mice. The protection was similar for the two mouse strains. In fact, there was actually a trend, albeit not statistically significant, toward improved survival in the eNOS-deficient mice. Multivariate analysis revealed that there was a significant effect on lethality of GT3 $(P=0.0001)$ and PTX $(P=0.002)$, but the presence/absence of eNOS did not have a significant effect. This clearly demonstrates that eNOS is not required for the protection against lethality by either drug or by the drug combination (Fig. 2).

\section{Intestinal Radiation Injury}

Intestinal radiation injury was assessed using intestinal mucosal surface area and crypt colony survival (Fig. 3). Radiation exposure induced a decrease in intestinal mucosal surface area at day 3.5 after radiation exposure. Both treatment with GT3 only and GT3 in combination with PTX improved postirradiation mucosal surface area $(P=0.002$ and $P=$ 0.0001 , respectively). A trend toward improved mucosal area was observed when treatment with GT3 combined with PTX was compared with treatment with GT3 only $(P=0.08)$. Treatment with PTX only did not affect postirradiation intestinal mucosal surface area. 
Treatment with GT3 and GT3 combined with PTX also improved intestinal crypt colony survival ( $P=0.0002$ and $P<0.0001$, respectively). No significant difference was observed between GT3 and GT3 together with PTX. Treatment with PTX only did not improve crypt colony survival.

\section{Hematopoietic Toxicity and Recovery}

Treatment with PTX, GT3 and GT3 in combination with PTX improved ex vivo colony formation from bone marrow harvested $24 \mathrm{~h}$ after $8.5 \mathrm{~Gy}$ TBI (Fig. 4). The effect of GT3 in combination with PTX was significantly more pronounced than the effect of treatment with PTX or GT3 only ( $P=0.005$ and $P=0.005$, respectively).

Spleen colony counts determined at day 10 after 8.5 Gy TBI showed a pattern similar to the ex vivo bone marrow CFU. PTX, GT3 and GT3 in combination with PTX increased spleen colony counts. The effect was most pronounced after the combination treatment. GT3 in combination with PTX significantly improved postirradiation spleen colony formation compared to GT3 only $(P=0.005)$.

Radiation induced a decrease in the numbers of circulating leukocytes, erythrocytes and platelets to a minimum at 10 days, after which blood cell counts partly recovered (data not shown). As reported before, GT3 treatment improved hematological recovery significantly. However, cotreatment with PTX did not affect circulating leukocyte and erythrocyte levels but did improve platelet recovery at day 14 after TBI $(P=0.05)$ (Fig. 4).

To obtain information about the bone marrow microenvironment, we determined cytokine levels in the supernatant of the medium used to collect bone marrow cells from the femurs. GT3 with or without PTX induced an increase in bone marrow plasma G-CSF at day $0(P=$ 0.005 and $P=0.005$, respectively) and day 1 postirradiation $(P=0.005$ and $P=0.005$, respectively).

As shown in Fig. 5, radiation exposure induced a decrease in bone marrow plasma IL-1a levels in the early postirradiation phase, with IL-1a levels below the detection limit of the assay at day 10 after TBI $(P=0.005)$. Treatment with GT3 in combination with PTX induced bone marrow plasma IL-1a levels at day 1 and day $10(P=0.004$ and $P=0.005$, respectively). Treatment with only GT3 does not increase IL-1a levels at day 1 ; however, it has an effect on day $10(P=0.008)$.

Radiation exposure induced a reduction in bone marrow plasma IL-6 to levels below the detection limit of the used assay (day 10: $P=0.005$ ). GT3 together with PTX increased the availability of IL-6 at day $1(P=0.01)$. GT3 did not affect IL-6.

Like IL-6, combination treatment also increased bone marrow plasma IL-9 levels at day 10 $(P=0.03)$.

GM-CSF levels were decreased after radiation exposure (day 1: $P=0.006$; day 10: $P=$ 0.008 ; day 14: $P=0.005$ ). None of the treatments affected the postirradiation GM-CSF levels.

\section{Vascular Peroxynitrite Production}

As shown before, GT3 treatment reduced postirradiation vascular peroxynitrite production $(P=0.02)$. This parameter did not appear to be affected by PTX (Fig. 6). Moreover, there was no difference between treatment with GT3 alone and GT3 combined with PTX. 


\section{DISCUSSION}

We have previously shown that a single dose of GT3 greatly reduces postirradiation intestinal, hematological and vascular changes as well as postirradiation lethality in mice with a DRF in excess of 1.3. Because PTX has been shown to enhance the effects of vitamin $\mathrm{E}$ when used in the prophylaxis or treatment of delayed effects of radiation in several organ systems, the aim of the present study was to investigate whether PTX could also enhance protection by GT3 against early TBI-induced toxicity and lethality.

PTX is a phosphodiesterase inhibitor developed to modify blood viscosity and to improve blood circulation. It has been on the market as a rheological agent for over 30 years. More recently, the agent's potential effects on cytokine-induced inflammatory responses, as well as other properties, have been recognized as well. PTX is believed to have beneficial effects in various inflammatory diseases, especially those with a TNF-a-driven inflammatory response.

Multiple studies have been performed to investigate the use of PTX as both a radioprotector and a radiosensitizer. Preclinical studies have shown that PTX might increase radiosensitivity of p53-mutated tumor cell lines by modulating cell cycle progression (1921). Moreover, PTX might suppress DNA double-strand break repair and improve tumor oxygenation $(22,23)$. When used as a tumor radiosensitizer, PTX does not appear to increase normal tissue radiation toxicity. In a phase III randomized clinical trial to study the effect of PTX on the radiation response of non-small cell lung cancer, PTX treatment did not increase dysphagia, odynophagia, pulmonary fibrosis or pneumonitis (24).

When studied as a radioprotector, PTX has often been used in combination with vitamin E. This combination therapy has been shown to reduce radiation fibrosis in various organ systems (4-9). Little is known about the effect of PTX on the acute radiation response. Ward et al. have shown that in rats PTX does not reduce (semi-) acute dermal and pulmonary radiation injury, assessed 2 months after radiation exposure (25).

As shown previously, GT3 reduces postirradiation intestinal injury. The current study shows that prophylactic treatment with PTX in combination with GT3 is more efficient in reducing TBI-induced mortality than treatment with PTX or GT3 alone. Our data suggest that the increased survival with the combination treatment is likely because of accelerated hematopoietic recovery and is not due to further improvement of gastrointestinal or vascular radiation responses. The addition of PTX to GT3 did not improve postirradiation crypt colony survival or intestinal mucosal surface area and failed to potentiate the effect of GT3 on postirradiation vascular peroxynitrite production. Rather, the additional protection conferred by PTX (in combination with GT3) in the "GI subsyndrome range" is assumed to be dependent on GI protection by GT3.

On the other hand, the combination therapy significantly increased both ex vivo bone marrow colony formation at 1 day after radiation exposure and in vivo spleen colony formation at day 10 postirradiation. Whereas combination treatment was shown to have a pronounced effect on postirradiation hematopoietic colony formation, only a modest effect on postirradiation circulating blood cell counts was observed in our experiment. Compared to GT3 alone, GT3 combined with PTX improved only platelet recovery; no effect on leukocytes or erythrocytes was observed over the benefit of GT3 alone. Although the lack of effect on peripheral leukocyte and erythrocyte recovery might seem unexpected considering the observed effect on bone marrow CFUs and spleen colonies, this observation might be explainable. First, GT3 is a potent radioprotector with strong hematopoietic effects. Our current survival data show that GT3 protects against TBI-induced death up to a dose of 11.5 Gy. Combination treatment was shown to be more potent, since it offered full protection 
against death even at a dose of $12.5 \mathrm{~Gy}$. In the current study, blood cell counts were performed after $8.5 \mathrm{~Gy}$ TBI. This dose was chosen to provide sufficient numbers of surviving animals in the vehicle- and PTX-treated groups, but it may not have been optimal for detecting a difference in peripheral blood cell recovery between the GT3 group and the GT3+PTX group. Moreover, the effect of adding PTX might be lineage specific and more pronounced for platelet formation than for the formation of leukocytes and erythrocytes.

To obtain information about the mechanism by which combination treatment modulates the bone marrow microenvironment and thereby the postirradiation hematopoietic response, we studied the effect of the different treatments on bone marrow plasma cytokine/chemokine levels. This technique to study the bone marrow niche was described by Kissel et al. (26). Cytokine/chemokine levels were expressed per mg bone marrow plasma protein. Notably, differences between treatment groups do not appear to be caused by differences in bone marrow cellularity. When cytokine/chemokines levels were expressed per $10^{7}$ present bone marrow cells, similar results were observed as when expressed per mg protein. When interpreting the bone marrow plasma data, it has to be borne in mind that PTX was administered only 30 min before radiation exposure and that the effect of PTX on the day 0 data might therefore be limited. Both GT3 and GT3 with PTX caused an increase bone marrow plasma G-CSF levels. Singh et al. have shown that GT3 treatment increases circulating G-CSF levels (27). They hypothesized that GT3 improves postirradiation hematopoietic recovery by stimulating the bone marrow with G-CSF. The fact the GT3 increases G-CSF levels in the bone marrow microenvironment may support this theory. However, because GT3 combined with PTX did not improve G-CSF levels compared with GT3 alone, the effects of combination treatment on the bone marrow niche appear to be regulated by factors different from G-CSF. On the other hand, since the experiments in the present study were performed at a sublethal radiation dose to permit serial measurements in control mice, it is also possible that the level of injury in GT3-treated animals was not sufficient to give rise to an increase in G-CSF levels.

Combined treatment with GT3 and PTX caused an increase in bone marrow plasma IL-1a, IL-6 and IL-9. All three interleukins are known to stimulate hematopoiesis (28-32). Administration of IL-6 has been shown to accelerate recovery from radiation-induced hematopoietic depression (29). Even though IL-6 is generally considered to be a multilineage stimulant, it may have a more pronounced effect on platelet recovery. For example, when administered after 5-fluorouracil treatment, IL-6 improves the recovery of circulating platelets, whereas no effect on circulating neutrophils is observed (30). IL-9 has been shown to enhance in vitro growth of both early erythroid and megakaryocytic progenitor cell colonies $(31,32)$. Considering the cytokine profile induced by GT3 with PTX and the observation that GT3 with PTX only improves postirradiation platelet recovery in the current experiment, it might be possible that the hematopoietic stimuli induced by GT3 with PTX are especially efficient in promoting megakaryocytic proliferation and platelet formation. While our data suggest that treatment with GT3 in combination with PTX affects hematopoietic recovery by the induction of hematopoietic stimuli like IL-1a, IL-6 and IL-9, further research is needed to study the effect of combined treatment on hematopoietic inhibitors. Lysofylline, a phosphodiesterase inhibitor like PTX, has been shown to suppress the release of hematopoietic inhibitors after treatment with cancer chemotherapeutic agents (33).

In the present study, bone marrow plasma studies were performed only until day 14 after TBI. Therefore, it is not possible to predict how long the observed increase in cytokines would persist or to what extent similar changes would be present systemically and influence the development of chronic changes in bone marrow or other organs. 
It was somewhat surprising that the protection against lethality from these drugs did not require the presence of eNOS. Many, if not all, pleiotropic effects of HMG-CoA reductase inhibitors are mediated through the eNOS pathway (10), and eNOS, through cAMP, also seems to play a prominent role in the mechanism of action of PTX. For example, both HMG-CoA (34) and PTX $(35,36)$ strongly upregulate thrombomodulin (TM), a potent natural anticoagulant on the endothelial cell surface, which appears to be involved in the regulation of normal tissue radiation responses (37). The finding that eNOS is not required for protection against lethality with either GT3 or PTX may suggest that the protection by these drugs is largely due to their properties as antioxidants and/or cytokine stimulators. On the other hand, it is also conceivable that HMG-CoA reductase is involved in the protection against lethality by non-eNOS-dependent mechanisms. Yet another possibility is that the role of eNOS after TBI is altered because of radiation-induced uncoupling, a process during which eNOS produces superoxide, rather than nitric oxide $(38,39)$. Further research is clearly needed to investigate the role of eNOS and microvascular oxidative/nitrosative stress in response to exposure to different radiation doses where radiation protectors such as GT3 do not confer complete protection. Such studies will allow for demonstration of partial eNOS dependence rather than an absolute requirement for this enzyme.

In conclusion, we have demonstrated that radio-prophylaxis with GT3 in combination with PTX is significantly more effective in improving survival after TBI than prophylaxis with GT3 alone. Our data suggest that administration of GT3 together with PTX may modulate the hematopoietic radiation response by the induction of hematopoietic stimuli. Combination therapy did not reduce postirradiation intestinal injury or vascular peroxynitrite production compared to treatment with GT3, and the protective effect does not appear to depend on eNOS.

\section{Acknowledgments}

We are grateful for assistance with tissue processing by Jennifer D. James of the Experimental Pathology Core Laboratory, Winthrop P. Rockefeller Cancer Institute, and performance of Luminex assays by Jeffrey C. Hale and Gregory D. Sempowski of the Immune Monitoring Core, Duke University Medical Center. Dr. Berbée was enrolled in the Ph.D. program at the Department of Radiation Oncology, University of Maastricht, the Netherlands. This work was supported by the National Institutes of Health (grants AI67798 and CA83719 to MH-J), the Defense Threat Reduction Agency (grants H.10027_07_AR_R and H.10045_07_AR_R to KSK), and the Veterans Administration.

\section{References}

1. Berbee M, Fu Q, Boerma M, Wang J, Kumar KS, Hauer-Jensen M. gamma-Tocotrienol ameliorates intestinal radiation injury and reduces vascular oxidative stress after total-body irradiation by an HMG-CoA reductase-dependent mechanism. Radiat Res. 2009; 171:596-605. [PubMed: 19580495]

2. Ghosh SP, Kulkarni S, Hieber K, Toles R, Romanyukha L, Kao TC, Hauer-Jensen M, Kumar KS. Gamma-tocotrienol, a tocol antioxidant as a potent radioprotector. Int J Radiat Biol. 2009; 85:598606. [PubMed: 19557601]

3. Kulkarni S, Ghosh SP, Satyamitra M, Mog S, Hieber K, Romanyukha L, Gambles K, Toles R, Kao TC, Kumar KS. Gamma-tocotrienol protects hematopoietic stem and progenitor cells in mice after total-body irradiation. Radiat Res. 2010; 173:738-747. [PubMed: 20518653]

4. Bese NS, Munzuroglu F, Uslu B, Arbak S, Yesiladali G, Sut N, Altug T, Ober A. Vitamin E protects against the development of radiation-induced pulmonary fibrosis in rats. Clin Oncol (R Coll Radiol). 2007; 19:260-264. [PubMed: 17433970]

5. Boerma M, Roberto KA, Hauer-Jensen M. Prevention and treatment of functional and structural radiation injury in the rat heart by pentoxifylline and alpha-tocopherol. Int $\mathrm{J}$ Radiat Oncol Biol Phys. 2008; 72:170-177. [PubMed: 18632215] 
6. Delanian S, Porcher R, Balla-Mekias S, Lefaix JL. Randomized, placebo-controlled trial of combined pentoxifylline and tocopherol for regression of superficial radiation-induced fibrosis. $\mathrm{J}$ Clin Oncol. 2003; 21:2545-2550. [PubMed: 12829674]

7. Delanian S, Porcher R, Rudant J, Lefaix JL. Kinetics of response to long-term treatment combining pentoxifylline and tocopherol in patients with superficial radiation-induced fibrosis. J Clin Oncol. 2005; 23:8570-8579. [PubMed: 16260695]

8. Misirlioglu CH, Demirkasimoglu T, Kucukplakci B, Sanri E, Altundag K. Pentoxifylline and alphatocopherol in prevention of radiation-induced lung toxicity in patients with lung cancer. Med Oncol. 2007; 24:308-311. [PubMed: 17873306]

9. Hille A, Christiansen H, Pradier O, Hermann RM, Siekmeyer B, Weiss E, Hilgers R, Hess CF, Schmidberger H. Effect of pentoxifylline and tocopherol on radiation proctitis/enteritis. Strahlenther Onkol. 2005; 181:606-614. [PubMed: 16170489]

10. Laufs U, La Fata V, Plutzky J, Liao JK. Upregulation of endothelial nitric oxide synthase by HMG CoA reductase inhibitors. Circulation. 1998; 97:1129-1135. [PubMed: 9537338]

11. Takemoto M, Liao JK. Pleiotropic effects of 3-hydroxy-3-methylglutaryl coenzyme A reductase inhibitors. Arterioscler Thromb Vasc Biol. 2001; 21:1712-1719. [PubMed: 11701455]

12. Kim NY, Pae HO, Kim YC, Choi CK, Rim JS, Lee HS, Kim YM, Chung HT. Pentoxifylline potentiates nitric oxide production in interleukin-1beta-stimulated vascular smooth muscle cells through cyclic AMP-dependent protein kinase A pathway. Gen Pharmacol. 2000; 35:205-211. [PubMed: 11827727]

13. Zhang XP, Tada H, Wang Z, Hintze TH. cAMP signal transduction, a potential compensatory pathway for coronary endothelial NO production after heart failure. Arterioscler Thromb Vasc Biol. 2002; 22:1273-1278. [PubMed: 12171787]

14. Zhang XP, Hintze TH. cAMP signal transduction induces eNOS activation by promoting PKB phosphorylation. Am J Physiol Heart Circ Physiol. 2006; 290:H2376-H2384. [PubMed: 16428343]

15. Baddeley AJ, Gundersen HJ, Cruz-Orive LM. Estimation of surface area from vertical sections. J Microsc. 1986; 142:259-276. [PubMed: 3735415]

16. Langberg CW, Sauer T, Reitan JB, Hauer-Jensen M. Relationship between intestinal fibrosis and histopathologic and morphometric changes in consequential and late radiation enteropathy. Acta Oncol. 1996; 35:81-87. [PubMed: 8619945]

17. Withers HR, Elkind MM. Microcolony survival assay for cells of mouse intestinal mucosa exposed to radiation. Int J Radiat Biol Relat Stud Phys Chem Med. 1970; 17:261-267. [PubMed: 4912514]

18. Kodell RL, Lensing SY, Landes RD, Kumar KS, Hauer-Jensen M. Determination of sample sizes for demonstrating efficacy of radiation countermeasures. Biometrics. 2010; 66:239-248. [PubMed: 19432769]

19. Theron T, Binder A, Verheye-Dua F, Bohm L. The role of G2-block abrogation, DNA doublestrand break repair and apoptosis in the radiosensitization of melanoma and squamous cell carcinoma cell lines by pentoxifylline. Int J Radiat Biol. 2000; 76:1197-1208. [PubMed: 10993631]

20. Waldeck W, Strunz AM, Muller G, Hotz-Wagenblatt A, Wijenne J, Langowski J, Didinger B, Debus J, Braun K. Induced and repressed genes after irradiation sensitizing by pentoxyphylline. Int J Cancer. 2007; 120:1198-1207. [PubMed: 17192923]

21. Strunz AM, Peschke P, Waldeck W, Ehemann V, Kissel M, Debus J. Preferential radiosensitization in $\mathrm{p} 53$-mutated human tumour cell lines by pentoxifylline-mediated disruption of the G2/M checkpoint control. Int J Radiat Biol. 2002; 78:721-732. [PubMed: 12194756]

22. Bohm L, Roos WP, Serafin AM. Inhibition of DNA repair by pentoxifylline and related methylxanthine derivatives. Toxicology. 2003; 193:153-160. [PubMed: 14599774]

23. Collingridge DR, Rockwell S. Pentoxifylline improves the oxygenation and radiation response of BA1112 rat rhabdomyosarcomas and EMT6 mouse mammary carcinomas. Int J Cancer. 2000; 90:256-264. [PubMed: 11091349]

24. Kwon HC, Kim SK, Chung WK, Cho MJ, Kim JS, Kim JS, Moon SR, Park WY, Ahn SJ, Na BS. Effect of pentoxifylline on radiation response of non-small cell lung cancer: a phase III randomized multicenter trial. Radiother Oncol. 2000; 56:175-179. [PubMed: 10927136] 
25. Ward WF, Kim YT, Molteni A, Ts'ao C, Hinz JM. Pentoxifylline does not spare acute radiation reactions in rat lung and skin. Radiat Res. 1992; 129:107-111. [PubMed: 1728051]

26. Kissel CK, Lehmann R, Assmus B, Aicher A, Honold J, Fischer-Rasokat U, Heeschen C, Spyridopoulos I, Dimmeler S, Zeiher AM. Selective functional exhaustion of hematopoietic progenitor cells in the bone marrow of patients with postinfarction heart failure. J Am Coll Cardiol. 2007; 49:2341-2349. [PubMed: 17572250]

27. Singh VK, Shafran RL, Jackson WE III, Seed TM, Kumar KS. Induction of cytokines by radioprotective tocopherol analogs. Exp Mol Pathol. 2006; 81:55-61. [PubMed: 16426603]

28. Fibbe WE, Willemze R. The role of interleukin-1 in hematopoiesis. Acta Haematol. 1991; 86:148154. [PubMed: 1785229]

29. Patchen ML, MacVittie TJ, Williams JL, Schwartz GN, Souza LM. Administration of interleukin-6 stimulates multilineage hematopoiesis and accelerates recovery from radiation-induced hematopoietic depression. Blood. 1991; 77:472-480. [PubMed: 1991164]

30. Takatsuki F, Okano A, Suzuki C, Miyasaka Y, Hirano T, Kishimoto T, Ejima D, Akiyama Y. Interleukin 6 perfusion stimulates reconstitution of the immune and hematopoietic systems after 5fluorouracil treatment. Cancer Res. 1990; 50:2885-2890. [PubMed: 2334892]

31. Donahue RE, Yang YC, Clark SC. Human P40 T-cell growth factor (interleukin-9) supports erythroid colony formation. Blood. 1990; 75:2271-2275. [PubMed: 1693525]

32. Fujiki H, Kimura T, Minamiguchi H, Harada S, Wang J, Nakao M, Yokota S, Urata Y, Ueda Y, Sonoda Y. Role of human interleukin-9 as a megakaryocyte potentiator in culture. Exp Hematol. 2002; 30:1373-1380. [PubMed: 12482498]

33. de Vries P, Singer JW. Lisofylline suppresses ex vivo release by murine spleen cells of hematopoietic inhibitors induced by cancer chemotherapeutic agents. Exp Hematol. 2000; 28:916923. [PubMed: 10989192]

34. Shi J, Wang J, Zheng H, Ling W, Joseph J, Li D, Mehta JL, Ponnappan U, Lin P, Hauer-Jensen M. Statins increase thrombomodulin expression and function in human endothelial cells by a nitric oxide-dependent mechanism and counteract tumor necrosis factor alpha-induced thrombomodulin downregulation. Blood Coagul Fibrinolysis. 2003; 14:575-585. [PubMed: 12960612]

35. Seigneur M, Dufourcq P, Belloc F, Lenoble M, Renard M, Boisseau MR. Influence of pentoxifylline on membrane thrombomodulin levels in endothelial cells submitted to hypoxic conditions. J Cardiovasc Pharmacol. 1995; 25(Suppl 2):S85-S87. [PubMed: 8699869]

36. Boldt J, Müller M, Heyn S, Welters I, Hempelmann G. Influence of long-term continuous intravenous administration of pentoxifylline on endothelial-related coagulation in critically ill patients. Crit Care Med. 1996; 24:940-946. [PubMed: 8681595]

37. Wang J, Zheng H, Ou X, Fink LM, Hauer-Jensen M. Deficiency of microvascular thrombomodulin and upregulation of protease-activated receptor 1 in irradiated rat intestine: possible link between endothelial dysfunction and chronic radiation fibrosis. Am J Pathol. 2002; 160:2063-2072. [PubMed: 12057911]

38. Landmesser U, Dikalov S, Price SR, McCann L, Fukai T, Holland SM, Mitch WE, Harrison DG. Oxidation of tetrahydrobiopterin leads to uncoupling of endothelial cell nitric oxide synthase in hypertension. J Clin Invest. 2003; 111:1201-1209. [PubMed: 12697739]

39. Berbee M, Fu Q, Kumar KS, Hauer-Jensen M. Novel strategies to ameliorate radiation injury: a possible role for tetrahydrobiopterin. Curr Drug Targets. 2010; 11:1366-1374. [PubMed: 20583982] 
A

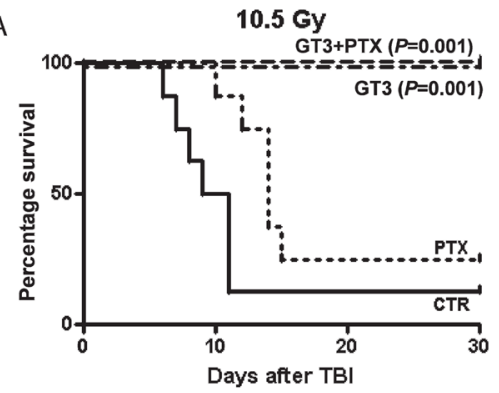

$12.5 \mathrm{~Gy}$

C

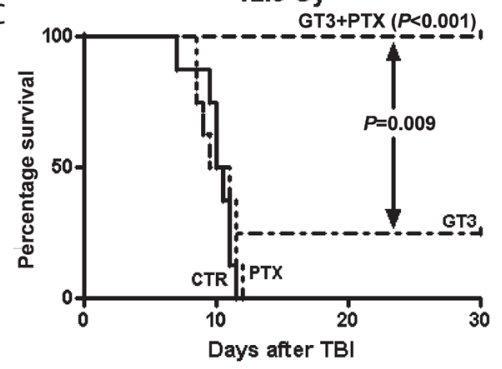

B

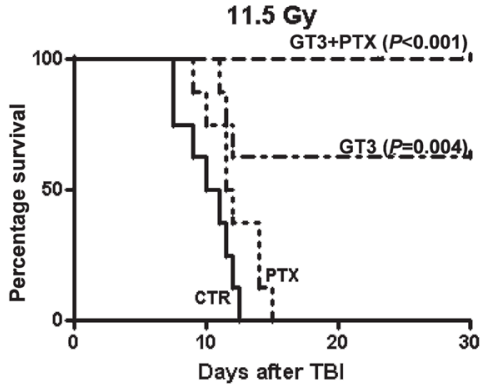

FIG. 1.

Kaplan-Meier survival curves for male CD2F1 mice after total-body irradiation. Mice were treated with 10.5 (panel A), 11.5 (panel B) or 12 Gy (panel C). PTX improved survival only in mice treated with 10.5 Gy. GT3 reduced lethality after both 10.5 and 11.5 Gy TBI.

Combination treatment prevented TBI-induced mortality up to $12.5 \mathrm{~Gy}$. At $12.5 \mathrm{~Gy}$, the combination therapy was significantly more effective than treatment with PTX or GT3 only. Eight animals per group from a single experiment. 

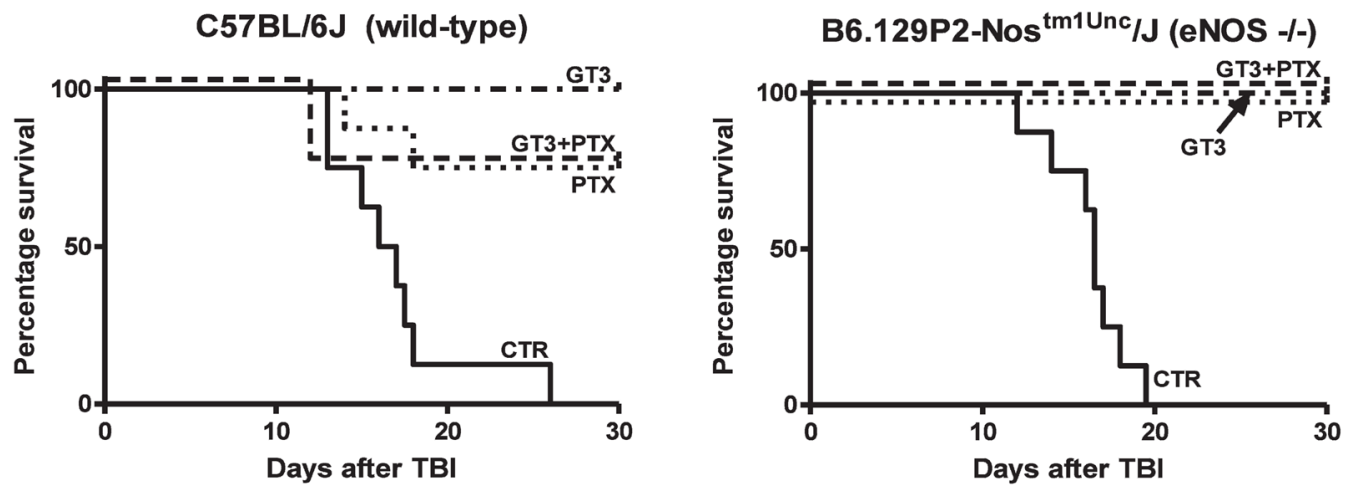

FIG. 2.

Kaplan-Meier survival curves for C57BL/6 mice (left panel) and B6.129P2-Nos ${ }^{\text {Tm1Unc }}$ mice (right panel) after $8.5 \mathrm{~Gy}$ total-body irradiation. Multivariate analysis revealed a significant effect of treatment with GT3 $(P=0.0001)$ and PTX $(P=0.002)$ but not an absolute requirement for eNOS after this dose of radiation. Eight animals per group from a single experiment. 

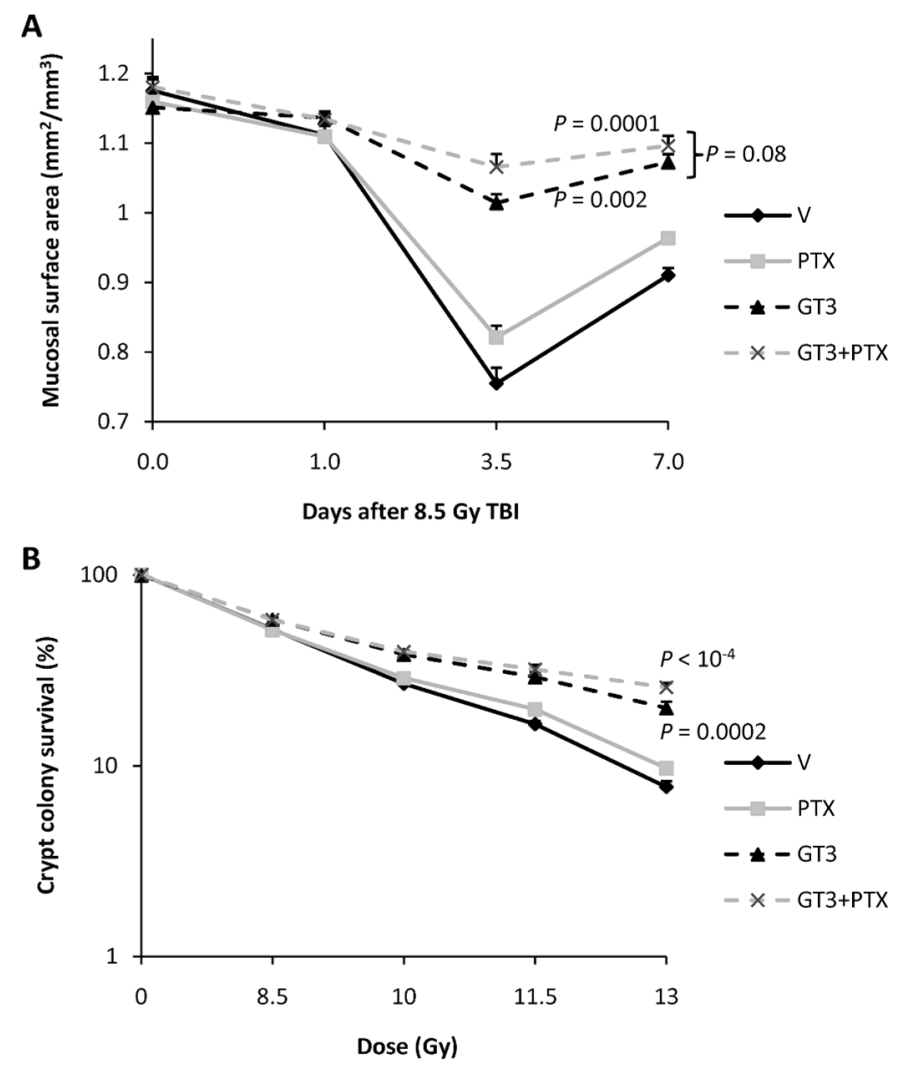

FIG. 3.

Panel A: TBI (8.5 Gy) induced a reduction in mucosal surface areas. Administration of a single dose of GT3 $24 \mathrm{~h}$ before irradiation significantly improved recovery. Combination treatment with GT3 and PTX did not significantly improve postirradiation mucosal surface area compared to treatment with GT3 only $(P=0.08)$. Treatment with PTX only did not affect mucosal surface area. Four animals per group from a single experiment (means \pm SEM). Panel B: Treatment with GT3 and GT3 combined with PTX improved intestinal crypt colony survival ( $P=0.0002$ and $P<0.0001$, respectively). No significant difference was observed between GT3 and GT3 together with PTX. Treatment with PTX only did not improve crypt colony survival. Six animals per group from a single experiment (means \pm SEM). 

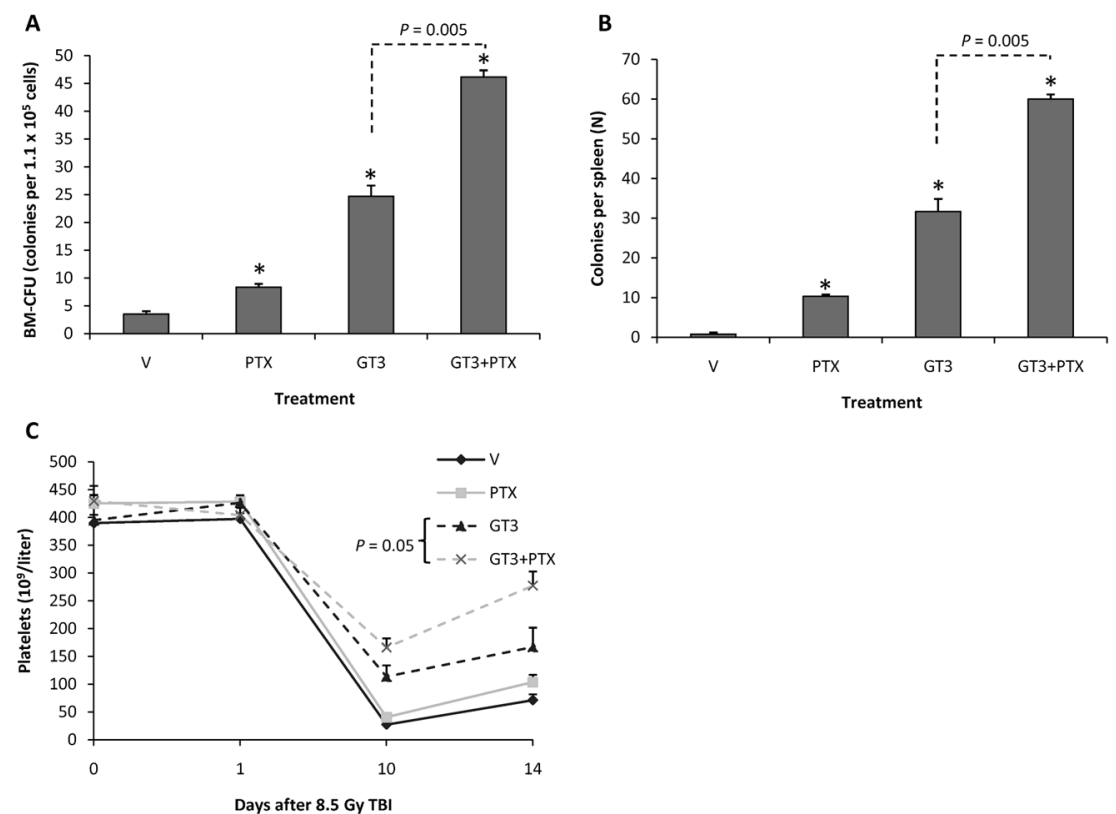

FIG. 4.

Panel A: Treatment with PTX, GT3 and GT3 in combination with PTX improved colonyforming units from bone marrow harvested $24 \mathrm{~h}$ after $8.5 \mathrm{~Gy}$ TBI. Combination therapy was significantly more effective than PTX or GT3 only ( $P=0.005$ and $P=0.005$, respectively). Six mice per group from a single experiment (means \pm SEM). Panel B: Treatment with PTX, GT3 and GT3 in combination with PTX improved spleen colony counts 10 days after TBI ( $8.5 \mathrm{~Gy})$. Combination therapy was significantly more effective than PTX or GT3 only $(P=$ 0.005 and $P=0.005$, respectively). Six mice per group from a single experiment (means \pm SEM). Panel C: Compared to treatment with GT3 only, combination therapy significantly improved platelet recovery at day 14 postirradiation $(8.5 \mathrm{~Gy})(P=0.05)$. Six mice per group from a single experiment (means \pm SEM). 
A
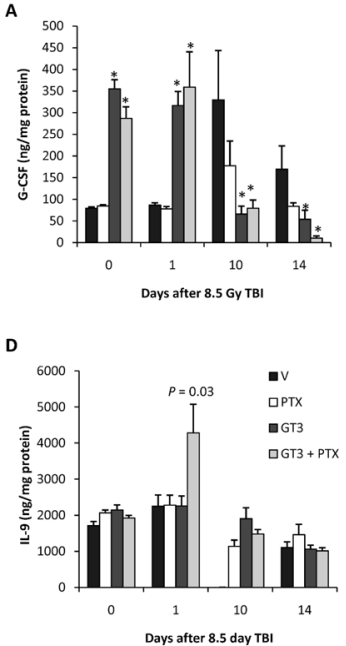
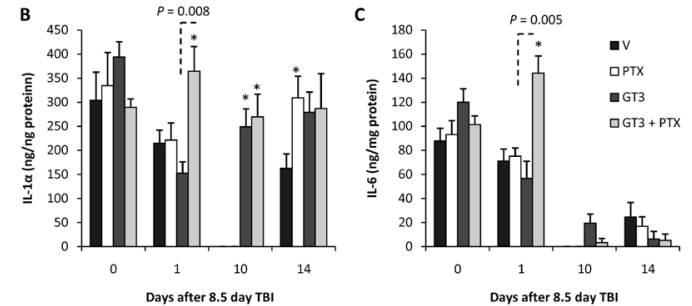

E

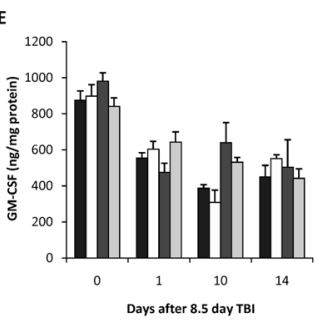

FIG. 5.

Panel A: Bone marrow plasma G-CSF levels after 8.5 Gy TBI. GT3, with or without PTX, induced an increase in bone marrow plasma G-CSF at day 0 and day 1 postirradiation. Panel B: Bone marrow plasma IL-1a levels after 8.5 Gy TBI. Treatment with GT3 in combination with PTX induced bone marrow plasma IL-1a levels at day 1 and day 10. Treatment with only GT3 did not increase IL-1a levels at day 1; however, it had an effect on day 10. Panel C: Bone marrow plasma IL-6 levels after 8.5 Gy TBI. IL-6 levels decreased after radiation exposure. GT3+PTX increased the availability of IL-6 at day 1 after TBI. GT3 did not affect IL-6. Panel D: Bone marrow plasma IL-9 levels after 8.5 Gy TBI. GT3+PTX increased the availability of IL-9 at day 1 after TBI. GT3 did not affect IL-9. Panel E: Bone marrow plasma GM-CSF levels after 8.5 Gy TBI. GM-CSF levels were decreased at day 1, 10 and 14 after radiation exposure. None of the treatments affected the postirradiation GM-CSF levels. Six mice per group from a single experiment (means \pm SEM). $* P<0.05$ compared to vehicle. 


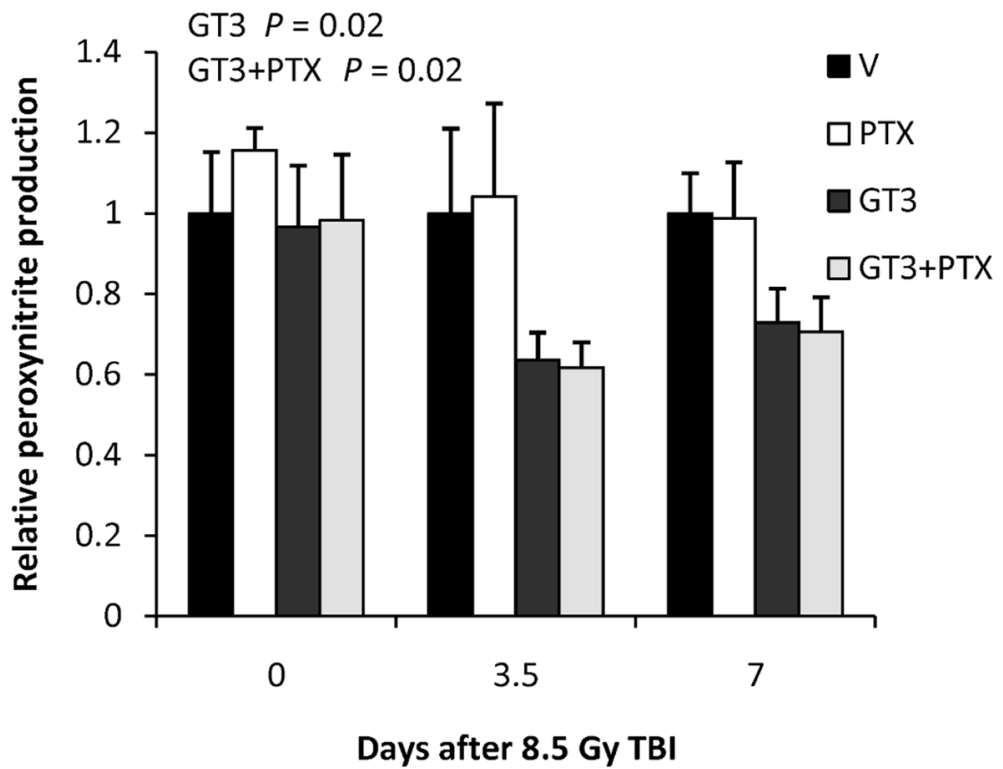

FIG. 6.

Treatment with GT3 and GT3 combined with PTX reduced postirradiation vascular peroxynitrite production $(P=0.02$ and $P=0.02$, respectively). No significant difference was observed between GT3 and GT3 together with PTX. Treatment with PTX only did not show an effect. Six mice per group from a single experiment (means \pm SEM). 
TABLE 1

Median Survival Times \pm Interquartile Range of Mice ( $N=8$ per group) after Total-Body Irradiation

\begin{tabular}{lccc}
\hline & $\mathbf{1 0 . 5}$ Gy & $\mathbf{1 1 . 5}$ Gy & $\mathbf{1 2 . 5} \mathbf{~ G y}$ \\
\hline Vehicle & $9(7-11)$ & $10(7.5-11.5)$ & $10(9.5-11)$ \\
Pentoxifylline & $14(12-15)$ & $11.5(1-14)$ & $9.5(8-11)$ \\
GT3 & $30(30-30)$ & $30(11.5-30)$ & $11(8.5-30)$ \\
GT3+PTX & $30(30-30)$ & $30(30-30)$ & $30(30-30)$ \\
\hline
\end{tabular}

